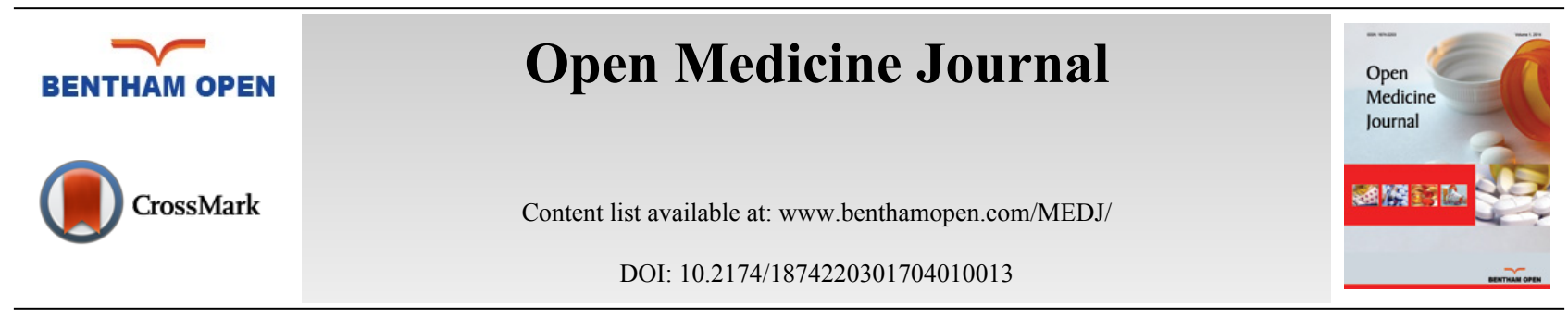

EDITORIAL

\title{
Some Perspectives on Quality Improvement in Healthcare
}

The notion of quality in healthcare has been a topic of writing and discussion since time immemorial. It is inextricably linked with the notion of what a good doctor or healthcare practitioner should do and as time has gone on, it has been linked with what a good hospital or primary care facility should do. Hippocrates laid down his basic precepts for quality in healthcare, beginning with primum non nocere, first do no harm. The refrain is echoed and developed by Osler in his Principles and Practice of Medicine whose every edition describes the relationship between the doctor and the patient and between the two of them and the disease or ailment [1]. Florence Nightingale developed the notion of quality in nursing care and created an image, which we take very much for granted today, of what a good quality hospital looks like: clean, organised, efficient in its use of resources [2]. Semmelweiss applied simple logic to determine why the rate of puerperal fever was so much greater in women tended by doctors than in women tended by midwives and, acting alone, fought to introduce the most simple procedures to improve patient care: hand washing after handling cadaveric material [3]. Semmelweiss's tale needs no rehearsal here. Suffice to say that his is an example of how struggling to improve quality in healthcare requires, on occasion, going diametrically against accepted wisdom and practice [4].

In the modern era, quality improvement in healthcare is driven forward on many fronts and for diverse motivations. The simple desire to see patients better cared for is, one hopes, the primary motivator but one needs to recognise other aspects: The costs of healthcare rise almost continuously, whether this be the cost of new drugs or instruments, the wage costs of personnel, the cost of litigation - an increasingly widespread phenomenon - the strains of the austerity and demands for cost-cutting which occur on a frequent basis, whether from government departments of health in those countries with a national health service, or insurance companies where healthcare is private, or the two together where there is a mixed economy of healthcare. Pressures exist from multiple directions to improve the quality of healthcare and this present issue of Open Medicine Journal seeks to discuss just a few of the directions in which quality improvement is taking place and to discuss just a few of the issues related to quality improvement in healthcare.

One need only do an online search is to for "quality improvement" and "healthcare" to see just how important this topic has become and just how much it is being discussed and debated. To get an idea of the range and diversity of topics which fall under quality improvement in healthcare, it suffices to glance at the abstracts from conferences from any of the several conferences on the topic that are held around the world each year.

The present thematic issue of Open Medicine Journal is therefore timely in its appearance and highly relevant in its range and diversity of topics, concerning as they do: Wikipedia and informal education in medical practice, simulation, quality management, ethics, personality type and mindfulness, language games in quality improvement and improving care in mental health through joint ventures between healthcare and the police. This is, of course, just a mere glimpse in terms of the range of possible topics but it is hoped that the very diversity will give a taste of what is going on in the field and in the literature.

\section{REFERENCES}

[1] Osler W. Principles and practice of medicine. D. Appleton and Co. New York: 1892.

[2] Nightingale F. Notes on Nursing: What it is and what it is not. Harrison, London 1860.

[3] Semmelweiss I, Ed. Etiology, Concept and Prophylaxis of Childbed Fever. University of Wisconsin Press; Madison, WI $1861 / 1983$.

[4] Roger LD, Ed. Asepsis or surgical cleanliness: A manual for physicians, students and nurses. Peoples Health Journal Co. Chicago 1900. 


\section{David Matheson (Guest Editor)}

Carnegie School of Education, Leeds Beckett University,

Leeds LS6 3QQ, UK

Tel: (+)44(0) 1138124704

E-mail: d.matheson@leedsbeckett.ac.uk

\section{(C) 2017 David Matheson.}

This is an open access article distributed under the terms of the Creative Commons Attribution 4.0 International Public License (CC-BY 4.0), a copy of which is available at: https://creativecommons.org/licenses/by/4.0/legalcode. This license permits unrestricted use, distribution, and reproduction in any medium, provided the original author and source are credited. 